\title{
Aharonov-Bohm oscillations and spin transport in a mesoscopic ring with a magnetic impurity
}

\author{
Sandeep K. Joshit, Debendranath Sahod ${ }^{-1}$ and A. M. Jayannavart \\ Institute of Physics, Sachivalaya Marg, Bhubaneswar 751 005, Orissa, India
}

(October 25, 2018)

\begin{abstract}
We present a detailed analysis of the Aharonov-Bohm (AB) interference oscillations manifested through transmission of an electron in a mesoscopic ring with a magnetic impurity atom inserted in one of its arms. The spin polarization transport is also studied. The electron interacts with the impurity through the exchange interaction leading to exchange spin-flip scattering. Transmission in the spin-flipped and spin-unflipped channels are explicitly calculated. We show that the entanglement between electron and spin-flipper states lead to a reduction of $\mathrm{AB}$ oscillations in spite of absence of any inelastic scattering. The spin-conductance (related to spin-polarized transmission coefficient) is asymmetric in the flux reversal as opposed to the two probe conductance which is symmetric under flux reversal. We point out certain limitations of this model in regard to the general notion of dephasing in quantum mechanics.
\end{abstract}

PACS Nos.: 73.23.-b, 05.60.Gg, 72.10.-d, 03.65.Bz

Quantum transport in open mesoscopic systems hasat. tracted considerable attention in the last two decadest 13 . In this area, the study of phase coherent transmission of electrons in the Aharonov-Bohm (AB) ring occupies a prominent place 6 . Study of dephasing and decoherence 11 of electrons in this geometry is very timely 12 to understand basic issues related to quantum phenomena. By introducing a magnetic impurity atom (to be referred to as the spin-flipper, or the flipper, for short) in one arm of the ring, one can couple the spin of the electron $(\vec{\sigma})$ to the spin of the flipper $(\vec{S})$ via the exchange interaction 1 . This leads to scattering of the electron in which the spin state of the electron and the impurity is changed without any exchange of energy. Additionally, this scattering leads to the entanglementinduced reduction of interference pattern 13 . Let the electron be incident from the left reservoir with its spin pointing "up" (see Fig. 1). The spin of the electron passing through the upper arm may or may not be flipped by the flipper. In the case that the spin is unflipped, one would expect the usual AB-oscillations of the transmission due to interference of the partial waves passing through the upper and the lower branches of the ring. However, in the case that the spin is flipped, one would think, guided by naive intuition, that a path detection has taken place and hence one would be led to conclude that the interference pattern for the spin-down component would be wiped out. This is true provided we consider only two forward propagating partial waves. However, there are infinitely many partial waves in this geometry which are to be superposed to get the total transmission. These arise due to the multiple reflections from the junctions and the impurity site. Consider, for example, an incident spin-up particle moving in the upper arm which is flipped at the impurity site and gets reflected to finally traverse the lower arm before being transmitted. Naturally, this par- tial wave will interfere with the spin-flipped component transmitted along the upper arm. This results in nonzero transmission for the spin-flipped electron. Thus on taking into account the multiple reflections (more than just two partial waves) the presence of magnetic impurity does not lead to "which-path" information. However, the presence of mafpetic impurity leads to the reduction of AB-oscillationstit. 13.

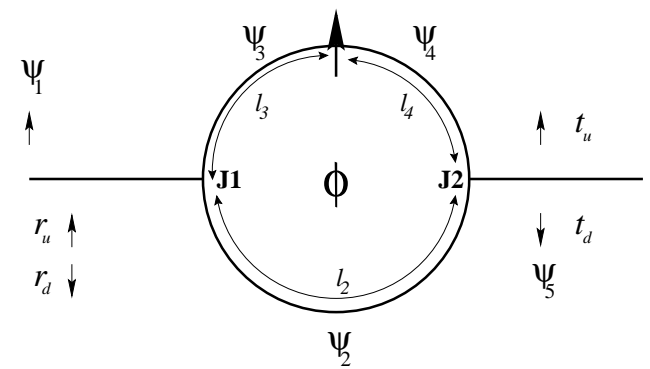

FIG. 1. Mesoscopic ring with Aharonov-Bohm flux $\phi$ threading through the center of the ring and a magnetic impurity in one arm of the ring.

In this work we study the reduction of amplitude of AB-oscillations arising due to the flipper. Within the same model we also study spin-polarized transport. There has been a great deal of interest in the concept of spintronic 14 , wherein, one manipulates with the spin of the charge carriers. Devices in which the electron spin stores and transmits information are being studied. Finally we discuss the limitations of this model (based on the interaction induced entanglement of quantum states) to the general understanding of dephasing in quantum systems. We study the problem using the quantum waveguide theory approach 6.15 and the spin degree of freedom of the electron is dealt with in line with Ref. 16. We consider an impurity consisting of a flipper capable of existing in $\mathrm{M}$ different discrete internal 
spin states and located at a particular position on the upper arm of the ring (see Fig. 11). The spin $\vec{\sigma}$ of the electron couples to the flipper spin $\vec{S}$ via an exchange interaction $-J \vec{\sigma} \cdot \vec{S} \delta\left(x-l_{3}\right)$. The magnetic flux threading the ring is denoted by $\phi$ and is related to the veg tor potential $A=\phi / l, l$ being the ring circumference 15 . During passage of the electron through the ring, the total spin angular momentum and its $z$-component remain conserved. We analyze the nature of spin-up/down and total transmission (reflection) coefficients. For this we consider the incident electron to be spin-polarized in the up-direction. We also show that up/down-transmission coefficients are asymmetric in flux reversal,i.e., total spin polarization (related to spin conductance 17 ) is asymmetric in flux reversal. As expected we find that the total transmission coefficient which is the sum of spin-up and spin-down transmission coefficients is symmetric in the flux reversal.

Let $l_{2}$ be the length of the lower arm of the ring and the impurity atom be placed at a distance $l_{3}$ from the junction $\mathrm{J} 1, l_{4}$ being the remaining segment length of the upper arm. The various segments of the ring and its leads are labeled as shown in Fig. 1 and the wave functions in these segments carry the corresponding subscripts. The wave functions in the five segments for a left-incident spin-up electron can be written as follows:

$$
\begin{aligned}
\psi_{1}= & \left(e^{i k x}+r_{u} e^{-i k x}\right) \chi_{m} \alpha+ \\
& r_{d} e^{-i k x} \chi_{m+1} \beta \\
\psi_{2}= & \left(A_{u} e^{i k_{1} x}+B_{u} e^{-i k_{2} x}\right) \chi_{m} \alpha+ \\
& \left(A_{d} e^{i k_{1} x}+B_{d} e^{-i k_{2} x}\right) \chi_{m+1} \beta, \\
\psi_{3}= & \left(C_{u} e^{i k_{1} x}+D_{u} e^{-i k_{2} x}\right) \chi_{m} \alpha+ \\
& \left(C_{d} e^{i k_{1} x}+D_{d} e^{-i k_{2} x}\right) \chi_{m+1} \beta, \\
\psi_{4}= & \left(E_{u} e^{i k_{1} x}+F_{u} e^{-i k_{2} x}\right) \chi_{m} \alpha+ \\
& \left(E_{d} e^{i k_{1} x}+F_{d} e^{-i k_{2} x}\right) \chi_{m+1} \beta, \\
\psi_{5}= & t_{u} e^{i k x} \chi_{m} \alpha+t_{d} e^{i k x} \chi_{m+1} \beta
\end{aligned}
$$

where $k_{1}=k+(e \phi / \hbar c l), k_{2}=k-(e \phi / \hbar c l), k$ is the wave-vector of incident electron. The wavefunction in Eq. 11 is a correlated function (entangled state) of the electron and the impurity spin which takes into account that the exchange interaction conserves the $z$-component of the total spin. The subscripts $u$ and $d$ represent "up" and "down" spin states of the electron with the corresponding spinors $\alpha$ and $\beta$ respectively (i.e., $\sigma_{z} \alpha=\frac{1}{2} \alpha$, $\sigma_{z} \beta=-\frac{1}{n} \beta$ ) and $\chi_{m}$ denotes the wave function of the impurity 16 with $S_{z}=m$ (i.e., $S_{z} \chi_{m}=m \chi_{m}$ ). The reflected (transmitted) waves have amplitudes $r_{u}\left(t_{u}\right)$ and $r_{d}\left(t_{d}\right)$ corresponding to the "up" and "down" spin components respectively. Contin and the current conservation $6,15,16$ at the junctions J1 and J2 imply the following boundary conditions.

$$
\begin{array}{r}
\psi_{1}(x=0)=\psi_{2}(x=0), \\
\psi_{1}(x=0)=\psi_{3}(x=0), \\
\psi_{1}^{\prime}(x=0)=\psi_{2}^{\prime}(x=0)+\psi_{3}^{\prime}(x=0),
\end{array}
$$

$$
\begin{array}{r}
\psi_{3}^{\prime}\left(x=l_{3}\right)-\psi_{4}^{\prime}\left(x=l_{3}\right)=G(\vec{\sigma} \cdot \vec{S}) \psi_{3}\left(x=l_{3}\right), \\
\psi_{3}\left(x=l_{3}\right)=\psi_{4}\left(x=l_{3}\right), \\
\psi_{4}\left(x=l_{3}+l_{4}\right)=\psi_{5}(x=0), \\
\psi_{2}\left(x=l_{2}\right)=\psi_{5}(x=0), \\
\psi_{2}^{\prime}\left(x=l_{2}\right)+\psi_{4}^{\prime}\left(x=l_{3}+l_{4}\right)=\psi_{5}^{\prime}(x=0) .
\end{array}
$$

Here $G=2 m J / \hbar^{2}$ is the coupling constant indicative of the "strength" of the spin-exchange interaction. The primes denote the spatial derivatives of the wave functions. Equations (1) along with the boundary conditions (2) were solved to obtain the amplitudes $t_{u}, t_{d}, r_{u}$ and $r_{d}$. Owing to the large length of the expressions in the following we confine ourselves to the graphical interpretation of the results. We have taken the flipper to be a spin-half object $(M=2)$ situated in the upper arm. Now, depending upon the initial state of the flipper we have possibility of either spin-flip scattering $\left(\sigma_{z}=1 / 2, S_{z}=-1 / 2\right)$ or no spin-flip scattering $\left(\sigma_{z}=1 / 2, S_{z}=1 / 2\right)$, as demanded by the conservation of the total spin and its $z$-component. In the case of nospin-flip scattering $\left(\sigma_{z}=1 / 2, S_{z}=1 / 2\right)$ the problem at hand reduces to that of simple potential scattering from the impurity. We have set $\hbar=2 m=1$ and throughout the value of interaction strength $G$ is given in dimensionless units. The parameters used for the analysis are mentioned in the figure captions.

To begin with we take a look at the symmetry properties of the transport coefficients in spin-flip scattering case where the electron spin is opposite to the flipper spin. It is worth noting that due to the presence of spin degree of freedom the problem in hand although onedimensional becomes a multichannel problem. Figure 2 shows the spin-up reflection coefficient $R_{u}=\left|r_{u}\right|^{2}$, spindown reflection coefficient $R_{d}=\left|r_{d}\right|^{2}$ and total reflection coefficient $R=R_{u}+R_{d}$ as a function of the magnetic flux parameter $\eta=\phi / \phi_{0}, \phi_{0}$ being the flux quantum $h c / e$ We clearly see the AB-oscillations with flux periodicity of $2 \pi \phi_{0}$. All three reflection coefficients are symmetric in the flux reversal as expected on general grounds 18 .

In Fig. 3 we plot the spin-up transmission coefficient $T_{u}=\left|t_{u}\right|^{2}$ (thin line), spin-down transmission coefficient $T_{d}=\left|t_{d}\right|^{2}$ (dashed line) and total transmission coefficient $T=T_{u}+T_{d}$ (thick line) versus $\eta$. It unambiguously shows that though the total transmission $T$ (related to the twoterminal electrical conductance) is symmetric in flux reversal the spin-up $T_{u}$ and spin-down $T_{d}$ components are asymmetric under flux reversal. These transmission coefficients show AB-oscillations with flux periodicity of $2 \pi \phi_{0}$. We have verified this behavior of the reflection and transmission coefficients for various values of wave vector $k$ of the incident electron and impurity strength $G$. These observations are consistent with the reciprocity relations for transport in multichannel systems 18 and are a consequence of the general symmetry properties of the Hamiltoniand. The transmission coefficient at flux $\phi$ for the case when the incident particle is spin-up and the 
impurity is spin-down is equal to the transmission coefficient for the case when incident particle is spin-down and impurity is spin-up but the flux direction is reversed. For the spin-polarized transport the totalpolarization $T_{u}-T_{d}$ is related to the spin-conductance 17 . The above symmetry properties imply that the spin-conductance is asymmetric under the flux reversal. This can be easily noted from Fig. 4. In the figure we have plotted the variation of spin polarization $\chi=\left(T_{u}-T_{d}\right) / T$ as a function of the magnetic flux $\phi$. This spin-polarization can be experimentally measured by using the well known spinvalve (magnetic valve or filter) effect 14 . In this method ferromagnetic metal pads are used at the junctions between the sample and the reservoirs. Using a ferromagnetic metal contact one can inject spin-polarized electrons. The transmitted electron intensity or current can be measured by tuning the magnetization axis of the ferromagnetic contact at the drain. This contact acts as a spin-polarizer. The current depends on the relative angle of polarization between the transmitted electrons and the ferromagnetic metal eqntact at the drain (wellknown $\cos ^{2}(\theta / 2)$ dependence) 14 . Our actual system may comprise of a single channel clean metallic ring and by doping it with a paramagnetic impurity atom 16 (or impurities 19 ) which has a virtual state in the continuum. It is also possible to use a quantum dot with one excess electron in one of the arm to replace the role of a magnetic impurity. It should be noted that at zero temperature the total electrical and spin conductances are to be calculated by summing up with equal weight-age the total transmission coefficients for all the four cases, i.e., $\sigma_{z}= \pm 1 / 2$ and $S_{z}= \pm 1 / 2$.

As discussed in the introduction, due to multiple reflections the presence of a spin-flipper in one arm does not lead to "which-path" information. This would have implied the complete blocking of spin-down transmission. In contrast we clearly observe the AB-oscillations for the case of $T_{d}$ originating from multiple reflections. We now address the question of partial loss of interference due to the spin-flipper. In Fig. 5 we have plotted the total transmission coefficient $T=T_{u}+T_{d}$ for the spin-flip scattering (SFS) case, and $T=T_{u}\left(T_{d}=0\right)$ for the no spin-flip scattering (NSFS) case for different parameters as indicated in the figures $5(\mathrm{a}-\mathrm{d})$. As expected $T$ exhibits $\mathrm{AB}$ oscillations which are periodic in flux with a period $\phi_{0}$ and they are symmetric under flux reversal. It is interesting to note, however, that the interference fringe visibility (or the magnitude of amplitude of $\mathrm{AB}$ oscillations) for the SFS case is always smaller than that for the case of NSFS. This clearly indicates partial decoherence.

To quantify the decoherence, we calculate the amplitude of $\mathrm{AB}$ oscillations by taking the difference between the maximum and the minimum of total transmission coefficient as a function of flux $\phi$ over one period of the oscillation. A plot of the variation of the amplitude of oscillation of total transmission $T$ with the interaction strength $G$ for the two cases, no spin-flip scattering (NSFS: $S=1 / 2 m=1 / 2$ ) and spin-flip scattering (SFS:
$S=1 / 2 m=-1 / 2$ ), are shown in figures Fig. 7 and 6 . The two figures correspond to two different locations of the impurity in the upper arm of the ring. Other parameters are mentioned in the respective figure captions. Note, however, the signature of loss of interference is that the amplitude of AB oscillation of transmission coefficient for the spin-flip case is always smaller than that for the no spin-flip case for all non-zero values of coupling strength $G$. In other words the reduction of amplitude of $\mathrm{AB}$ oscillations is stronger for the spin-flip scattering case. We have verified the above observation for other parameters in the problem. Thus the presence of spinflipper reduces the AB-oscillations. This substantiates our claim of decoherence due to entanglement.

At this point we are inclined to think that the harmonic components of the total transmission $T(\eta)$ in $\eta=\phi / \phi_{0}$ might be able to shed more light on the issue. So, with the hope of extracting some systematics we plot the $n^{t h}$ harmonic component $a_{n}=\int_{0}^{2 \pi} T(\eta) \cos (n \eta) d \eta$ for $n=1,2,3 \ldots$ as a function of strength $G$ for the spin-flip scattering as well as no spin-flip scattering cases. The plots are shown in Fig. \& for first four harmonic components. As can be seen the harmonic components do not show any systematics in the sense that the higher harmonic components can dominate over the lower harmonic components at certain values of strength $G$ for spin-flip scattering as well as no spin-flip scattering cases. Also, the $n^{\text {th }}$ harmonic component for spin-flip scattering could dominate over that of the no spin-flip scattering component. These features of the harmonic components are manifestations of the multiple scattering nature of the transport in such ballistic systems as against the observation of domination of lowest harmonic component $(n=1)$ in the case of transport in the presence of evanescent modes 20 . Guided by the naive intuition mentioned earlier we would have expected the lowest harmonic to dominate. This reiterates the important role played by the reflection at the impurity site. We would like to emphasize that irrespective of the behavior of the harmonic components (say for a particular case $n^{\text {th }}$ harmonic component in the spin-flip case is dominant over the same $n^{\text {th }}$ harmonic component for no-spin-flip case) the AB-oscillations of the total transmission are always suppressed in the spin-flip case.

In order to make sure that nothing unusual happens at other energies we study the $T, T_{u}$ and $T_{d}$ as functions of $k l$ for the case of spin-flip scattering. Figure 9 reveals an interesting fact, namely at $k l=2 \pi+4 n \pi, n=0,1,2 \ldots$ the $T_{d}$ component vanishes independent of the value of interaction strength $G$. In the $\eta \neq 0$ case this happens at $k l=4 n \pi, n=1,2, \ldots$. At these values of the incident wave-vectors the electron wave function at the impurity site happens to be zero. As a result the electron does not interact with the impurity at all and consequently there is no spin-flip scattering at these energies. However, these k-points are to be distinguished from those at which although $T_{d}$ is zero but in addition $T_{u}=1$, be- 
cause at these resonant energies the restriction $T+R=1$ forces $T_{d}, R_{u}$ and $R_{d}$ to be zero.

In our separate study 21 we have analysed the same model in regard to current magnification effect22 25 which is also a purely quantum phenomenon. Against our naive intuition we find that in some parameter regime spin-flip scattering (or entanglement) enhances the magnitude of the current magnification as opposed to the suppression of the AB-oscillations. According to the general notion of dephasing or decoherence one expects all typical features of quantum mechanical probability effects to be suppressed. In full generality dephasing can be defined as the phenomenon by which quantum mechanical systems behave as though they are described by classical probability theory. Only the presence of inelastic scattering or coupling the system to infinite environmental degrees of freedom (bath), leading to irreversible loss of phase memory, can dephase both AB-oscillations and reduce current magnification effect simultaneously. We believe that the suppression of some quantum features and non-suppression other quantum effects is a characteristic of entanglement and the absence of inelastic scattering. We expect the same in other models based only on the notion of entanglement. Moreover, let us emphasize that in our model the environment consists of a single atom only.

In conclusion, we have studied in detail the nature of reduction of $\mathrm{AB}$-oscillations in mesoscopic ring in the presence of a spin-flipper in one of its arms. The presence of magnetic impurity makes the polarized transmission coefficient asymmetric in flux reversal whereas the total transmission coefficient is symmetric in line with the theoretical expectations. We have also pointed out the limitations of this entanglement based model in describing the phenomenon of dephasing in quantum systems. Further case of spin-flipper with higher number of internal states and spin-flippers in both arms of the ring are under investigation.

\section{ACKNOWLEDGMENTS}

One of us (DS) would like to thank Professor S. N. Behera for extending hospitality at the Institute of Physics, Bhubaneswar.
${ }^{2}$ S. Datta, Electronic transport in mesoscopic systems (Cambridge University Press, Cambridge, 1995).

${ }^{3}$ P. S. Deo and A. M. Jayannavar, Pramana J. Phys. , (2000) in print, preprint cond-mat/0006035.

${ }^{4}$ S. Washburn and R. A. Webb, Adv. Phys., 35 (1986) 375.

${ }^{5}$ Y. Gefen, Y. Imry, and M. Ya. Azbel, Phys. Rev. Lett. 52, 129 (1984).

${ }^{6}$ A. M. Jayannavar and P. S. Deo, Phys. Rev. B 49, 13685 (1994); 51, 10175 (1995); P. S. Deo and A. M. Jayannavar, Phys. Rev. B 50, 11629 (1994);

${ }^{7}$ A. Stern, Y. Aharonov and Y. Imry, Phys. Rev. A 41, 3436 (1990).

${ }^{8}$ P. Mello, Y. Imry and B. Shapiro, Phys. Rev. B 61, 16570 (2000).

${ }^{9}$ M. Büttiker, Phys. Rev. B 33, 3020 (1986);

10 T. P. Pareek, S. K. Joshi and A. M. Jayannavar, Phys. Rev. B 57, 8809 (1998).

11 T. P. Pareek, A. M. Jayannavar and N. Kumar, Ind. J. Phys., (2000) in print, cond-mat/9811368.

12 D. Sprinzak, E. Buks, M. Heiblum and H. Shtrikman, Phys. Rev. Lett. 84, 5820 (2000) and references therein.

${ }^{13}$ L. S. Schulman, Phys. Lett. A 211, 75 (1996), and references therein.

${ }^{14}$ G. Prinz, Phys. Today 48, 58 (1995); Science 282, 1660 (1998) and references therein.

15 J-B. Xia, Phys. Rev. B 45, 3593 (1992).

${ }^{16}$ O. L. T. de Menezes and J. S. Helman, Am. J. Phys. 53, 1100 (1985).

17 S. Das Sarma, J. Fabian, X. Hu, I. Zutic, Superlattices and Microstructures 27, 289 (2000); I. Zutic and S. Das Sarma, Phys. Rev. B 60, 16322 (1999).

18 M. Büttiker, IBM J. Res. Dev. 32, 317 (1988).

19 A. Vedyeyev et. al., Phys. Rev. B 63, 64429 (2001).

${ }^{20}$ A. M. Jayannavar and P. S. Deo, Mod. Phys. Lett. B 8, 301 (1994); B. C. Gupta, P. S. Deo and A. M. Jayannavar, Int. J. Mod. Phys. B 10, 3595 (1996).

${ }^{21}$ Colin Benjamin, Sandeep K. Joshi, Debendranath Sahoo and A. M. Jayannavar, unpublished, preprint condmat/001140\%.

${ }^{22}$ A. M. Jayannavar and P. S. Deo, Phys. Rev. B 51, 10175 (1995);

23 T. P. Pareek, P. S. Deo and A. M. Jayannavar, Phys. Rev. B 52, 14657 (1995).

${ }^{24}$ M. V. Moskalets, Euro. Phys. Lett. 41, 189 (1998).

${ }^{25}$ T. Choi, C. M. Ryu and A. M. Jayannavar, Int. J. Mod. Phys. B 12, 2091 (1998); preprint cond-mat/9808245. $\dagger$ Electronic address: joshi@iopb.res.in

¥ Permanent address: Materials Science Division, IGCAR, Kalpakkam 603 102, Tamil Nadu, India. Electronic address: dsahoo@iopb.res.in

* Electronic address: jayan@iopb.res.in

${ }^{1} \mathrm{Y}$. Imry, Introduction to Mesoscopic Physics (Oxford University, New York, 1997). 


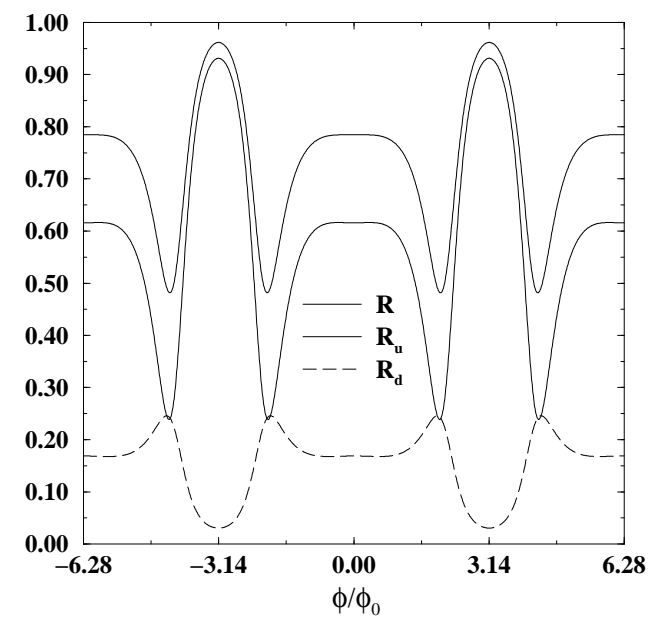

FIG. 2. Plot of total reflection coefficient $R$, spin-up reflection coefficient $R_{u}$ and spin-down reflection coefficient $R_{d}$ for the spin -flip scattering case. The parameters are $k l=1.0$, $G=10.0$.

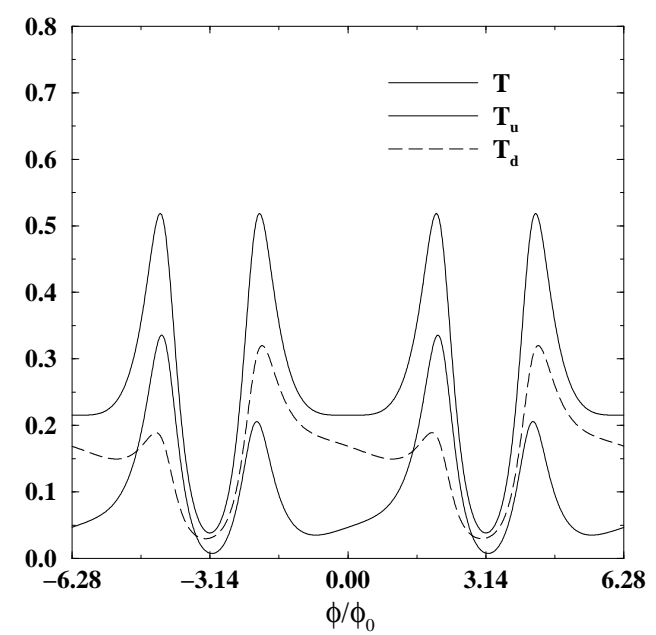

FIG. 3. Plot of total transmission coefficient $T$, spin-up transmission coefficient $T_{u}$ and spin-down transmission coefficient $T_{d}$ for the spin -flip scattering case. The parameters are $k l=1.0, G=10.0$.

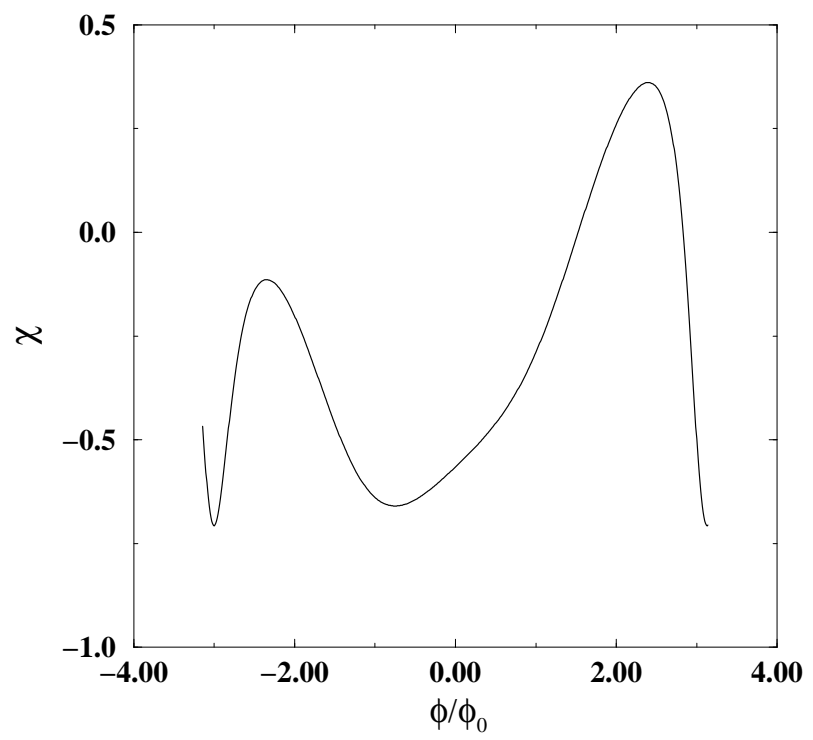

FIG. 4. Spin polarization $(\chi)$ as a function of the flux $\phi$ for interaction strength $G=10.0$.

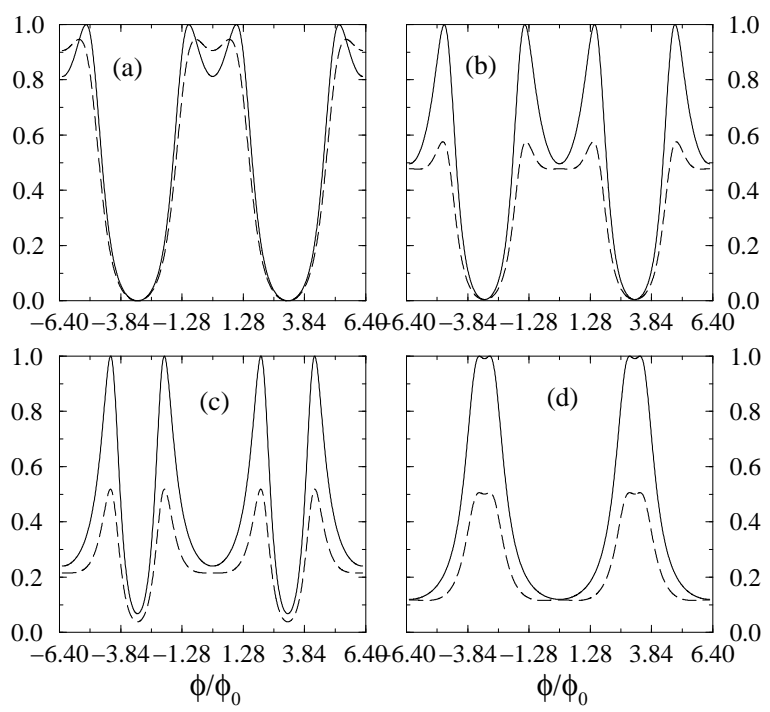

FIG. 5. Amplitude of AB oscillations or interference fringe visibility for the two cases of SFS and NSFS for different strengths of the exchange interaction. In all four cases $l_{2} / l=0.5, l_{3} / l=l_{4} / l=0.25$ and $k l=1.0$. The values of coupling strength $G$ are (a) $G=1.0$, (b) $G=5.0$, (c) $G=10.0$ and (d) $G=15.0$. 


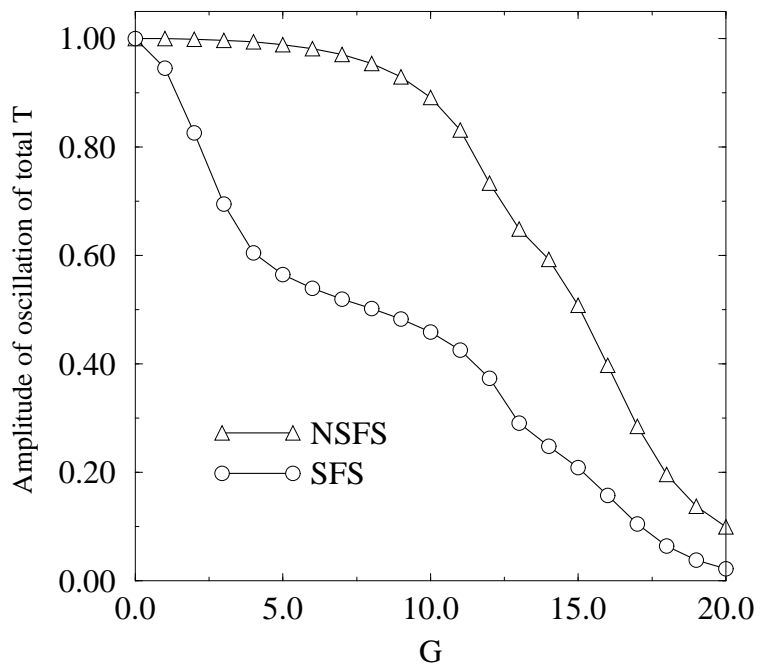

FIG. 6. Variation of Amplitude of AB oscillations with increasing strength $G$ of spin-flipper for the case of asymmetrically placed flipper. $l_{3} / l=0.15, l_{4} / l=0.35$ and $k l=1.0$.

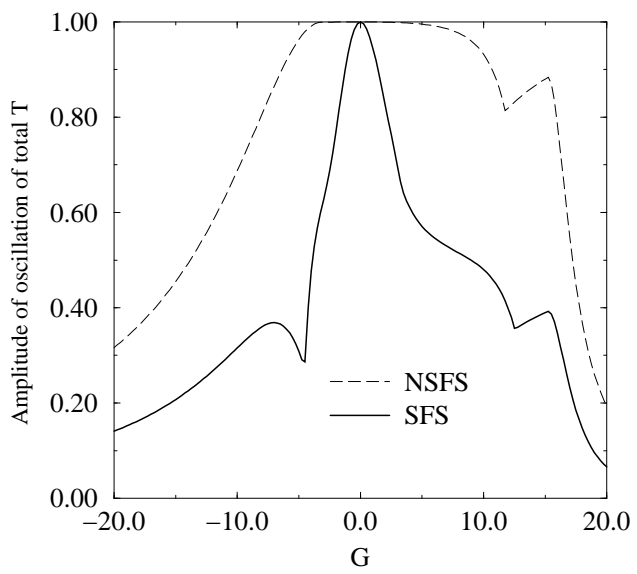

FIG. 7. Variation of amplitude of oscillation of total transmission coefficient with the interaction strength for the two cases of flip and no-flip scattering. The parameters are $k l=1.0$.

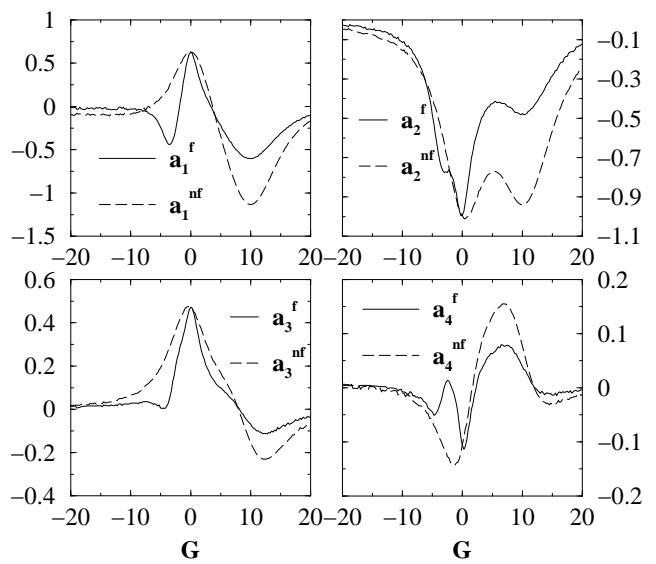

FIG. 8. Variation of $n^{\text {th }}$ harmonic component $a_{n}$ of the total transmission coefficient with the interaction strength $G$ at $k l=1.0$. Dashed lines are for the no-flip case and solid lines are for the flip case.
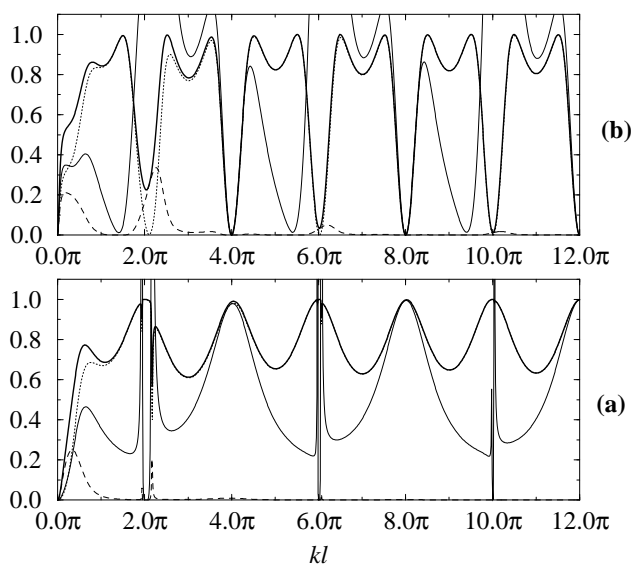

FIG. 9. The transmission spectrum of $T$ (thick line), $T_{u}$ (dotted line) and $T_{d}$ (dashed line) for (a) $\eta=0.0 G=5.0$ and (b) $\eta=1.3, G=5.0$. Thin line shows the plot of the unnormalized electron probability $\left|\psi_{3}\left(l_{3}\right)\right|^{2}$ at the impurity site $x=l 3$. 